\title{
The TNF Egr participates in signaling during cell competition in the absence of a requirement for JNK
}

\author{
Albana Kodra ${ }^{1}$, Claire de la Cova ${ }^{2}$, Aditi Sharma Singh ${ }^{1}$ and Laura A. Johnston ${ }^{1 \uparrow}$ \\ ${ }^{1}$ Department of Genetics \& Development, Vagelos College of Physicians and Surgeons, \\ Columbia University, New York, NY. \\ ${ }^{2}$ Department of Biological Sciences, University of Wisconsin, Milwaukee, WI. \\ ^ corresponding author: lj180@columbia.edu
}

\begin{abstract}
Numerous signaling pathways have been implicated in the elimination of cells in cell competition. Here we explore the relative contributions of two of them, the recently discovered CCSM and the conserved JNK stress pathway, using a series of genetic interactions tests. We demonstrate that the expression of the TNF Eiger (Egr), and the TNFR adaptor protein Traf4, are specifically up-regulated in the wild-type "loser" cell population during Myc-mediated cell competition. We find that the absence of Egr or its receptor Grindelwald (grnd) robustly prevents elimination of the loser cells in genetic cell competition assays. In contrast, although canonical JNK signaling is activated downstream of these adaptors, loss of either of the JNK effectors Tak1/JNKK or Hemipterous/JNK is not sufficient to prevent loser cell elimination in the competitive context. Our results instead suggest that Egr/Grnd influences the Rel activator Dredd in carrying out the cells' competitive death and elimination. Our experiments thus provide evidence that although Egr/Grnd signaling activates two parallel pathways in Myc cell competition, only the CCSM is sufficient to remove the wild-type loser cells from the tissue.
\end{abstract}

\section{Introduction}

Cell competition is an evolutionarily conserved cell selection process, based on comparisons of relative cellular fitness, that allows the healthiest cells ("winners") to colonize a growing tissue while causing relatively less fit (but otherwise viable) cells to die and be eliminated from the epithelium ("losers") (JOHNSTON 2009). The specific mechanisms that trigger elimination of the loser cells are influenced by the tissue type, the particular circumstances of the competition and the genetic background. Genetic 
studies of cell competition in the imaginal discs of Drosophila have demonstrated context-dependent deployment of several highly conserved signaling pathways, that almost always includes activation of the stress response Jun $\mathrm{N}$-terminal kinase (JNK) pathway (MORENo et al. 2002a; DE LA COVA et al. 2004; MORENO AND BASLER 2004; TYLER et al. 2007; IGAKI et al. 2009; OHSAWA et al. 2011; BANRETI AND MEIER). In addition, a novel cell competition signaling module (CCSM) consisting of select components from the immune deficiency (IMD) and Toll immune response pathways that functions in some contexts of cell competition was recently identified (MEYER et al. ; AlPAR et al. 2018; NAGATA et al. ; HOF-MiCHEL et al.).

Cell competition operates by an instructive mechanism through which information is exchanged between two cell populations that results in apoptosis of the relatively weaker cells. In this sense, cell competition resembles the highly conserved tumor necrosis factor (TNF) superfamily-driven extrinsic cell death pathway, utilized in immunity and inflammation (NAGATA 1997). The sole Drosophila TNF, Eiger, has homology with the mammalian TNF, Ectodysplasia (EDA)-A2, and many human TNF ligand superfamily proteins (e.g. RANKL, CD40L, FasL, TNF- $\alpha$, and TRAIL) (IGAKI et al. 2002). Like other TNF proteins, Egr is a type II transmembrane protein but can also be cleaved and glycosylated, allowing it to be secreted (KAUPPILA et al. 2003). Egr binds to the Drosophila TNF Receptors (TNFRs) Wengen (Wgn) (KANDA et al. ; KAUPPILA et al.) and Grindelwald (Grnd) (ANDERSEN et al. 2015), and the Egr/TNFR complex functions by activating the conserved core kinase cascade of the c-Jun $\mathrm{N}$-terminal kinase (JNK) pathway that includes the Tak1 MAP3K/JNKK kinase, the Hemipterous (Hep) MAP2K/JNK kinase, and the Basket (Bsk) MAPK/JNK (KANDA et al. ; KAUPPILA et al. 2003; ANDERSEN et al. 2015).

In the immune response, Egr/TNFR signaling cooperates with the IMD pathway to activate expression of anti-microbial peptides (AMPs) in the fat body (FB), an adipose tissue and major immune organ in Drosophila (DELANEY et al. 2006). Upon infection, Egr/TNFR activation leads to Tak1 activity, activating JNK signaling; in parallel, Tak1 is independently activated by the IMD pathway, thereby regulating the activity of the NFkB, Relish (Rel) (DELANEY et al. 2006; KLEINO AND SILVERMAN). Rel activation requires proteolytic cleavage by the caspase 8 homolog Dredd, yielding the active $\mathrm{N}$-terminal domain, Rel68 (STOVEN et al. 2003). Dredd's cleavage of Rel occurs upstream of Tak1 (ZHOU et al. 2005), but Tak1 is also separately required in a downstream function to activate the I-kappaB kinase (IKK) complex. In turn, the IKK complex phosphorylates 
Rel, permitting it to activate AMP gene expression (ERTURK-HASDEMIR et al. 2009). Expression of Dredd is sufficient to induce Rel cleavage in S2 cells (STOVEN et al. 2000; StOVEn et al. 2003; ERTURK-HASDEMIR et al. 2009; PAQUETTE et al. 2010), and in some contexts Dredd expression can induce cell death (HU AND YANG ; CHINCHORE et al. 2012; KIM et al. 2014; MEYER et al. 2014). Interactions between JNK signaling and the Toll immune pathway have also been described (Wu et al. 2015).

In contrast to the canonical IMD and Toll immune response pathways, the CCSM appears to consist of a few components from each of these pathways, and includes Toll and several other Toll related receptors (TRRs), the secreted Toll ligand Spätzle (Spz), the caspase Dredd, and the Rel NF-kB (MEYER et al. ; ALPAR et al.). In competitive interactions between WT cells and cells that express an extra copy of the conserved transcriptional regulator Myc (hereafter called Myc competition), the CCSM is activated via the locally increased production and activation of Spz by Myc-expressing cells. Spz activation, which requires cleavage by the serine protease SPE, in turn leads to Toll activity in nearby wild type (WT) cells, causing them to acquire a "loser" fate, and activating Rel and expression of the hid gene, encoding a proapoptotic factor (ALPAR et al.). CCSM signaling culminates in the death and elimination of the loser cells from the growing wing disc epithelium (MEYER et al. ; ALPAR et al. 2018). Although the CCSM is related to the IMD and Toll immune response pathways and employs several of their components, their outcomes are intriguingly distinct. First, the CCSM leads to the killing of the relatively less fit "loser" cells in actively proliferating tissues such as wing discs, but it does not activate AMP gene expression, nor is it activated in immune tissues like the FB (MEYER et al. 2014; ALPAR et al. 2018). Second, it requires only some of the factors from each of the canonical IMD and Toll pathways. For example, SPE, Spz, Toll and other TRRs are required to kill loser cells in cell competition, but the conserved adaptor MyD88 and the Pelle kinase are both dispensable (MEYER et al. 2014). From the IMD pathway, the function of FADD and Dredd are necessary to eliminate loser cells, but IMD itself is not required, nor is Tak1 or the IKK complex. However, Rel activity is critical for loser cell death, as is Dredd; genetic loss of either prevents apoptosis of the loser cells (MEYER et al. 2014). The CCSM thus activates a genetic program that is apparently quite distinct from that of the canonical immune response (MEYER et al. 2014; ALPAR et al. 2018). Importantly, the CCSM is activated locally and contained within the tissue in which WT and Myc-expressing cells are competing; unlike the immune response, it is not activated systemically (ALPAR et al.). 
The JNK stress pathway is also activated during Myc cell competition in wing discs. Expression of puc-lacZ, a transcriptional reporter of the JNK target gene, puckered, can be variably activated in loser and winner cells (DE LA COVA et al. ; MORENO AND BASLER). However, the importance of JNK pathway activity in the elimination of the loser cells in Myc competition has remained unclear. In one report, only $30 \%$ of loser cell death was suppressed by the absence of JNK signaling (DE LA COVA et al.). In other work, suppression of loser cell death by blocking JNK also required expression of p35, a pancaspase inhibitor (MORENO AND BASLER). By contrast, Myc competition can be completely suppressed by the genetic loss of any component of the CCSM.

With the goal of shedding light on the relative contributions of the JNK and CCSM signaling pathways to the death of the loser cells, we explored genetic interactions between them. We show here that egr expression, and that of Traf4, encoding a TNFR adaptor protein, are specifically up-regulated in WT "loser" cell population during Mycmediated competition. We find that the absence of egr, or its receptor grnd, robustly prevents elimination of the loser cells in cell competition assays. Genetic experiments indicate that Egr/Grnd activity is transduced via Traf4 and Traf6 and results in the activation of the JNK pathway in the loser cells. However, our results reveal that canonical JNK signaling downstream of these adaptors is not sufficient for loser cell elimination in the competitive context: genetic experiments indicate that Tak1 and the downstream JNK kinase Hep are dispensable, and a dominant negative form of the kinase Bsk only partially suppresses their competitive elimination. Although Egr/Grnd activity in the WT loser cells is largely independent of JNK activity, our results point to their influence on the Rel activator Dredd in carrying out the cells' competitive death and elimination. Our experiments thus provide evidence that Egr/Grnd signaling activates two parallel pathways in Myc stimulated cell competition, only one of which - the CCSM - is sufficient to remove the WT loser cells from the tissue.

\section{Materials and Methods}

Fly strains and Husbandry. Flies were raised at $25^{\circ} \mathrm{C}$ on standard cornmeal-molasses food supplemented with fresh dry yeast. The following strains were used: hep ${ }^{r 75}$, grnd $^{\text {M105292 }}$, grnd ${ }^{\text {DfBSC149 }}$, Tak11 ${ }^{1}, T_{a k}{ }^{2}, U A S-B s k^{D N}$, and act>y+>Gal4 (from Bloomington Drosophila Stock Center, BDSC); $w g n^{22}$ (gift of P. Barker), egr ${ }^{3 A G}$, made from outcrossing egr ${ }^{3}$ to rid the chromosome of NimC1 mutations (KODRA et al.); UAS-Grnd$I R^{K K}$ (Vienna Drosophila Resource Center, VDRC); UAS-egr-IR and UAS-dTRAF4IR 
(gifts of M. Miura); UAS-egr ${ }^{\# 5} / C y O$ and UAS-dTRAF6 ${ }^{I R}$ (gifts of T. Igaki); UAS-grnd ${ }^{\text {extra }}$ and UAS-grndintra (gifts of P. Leopold); UAS-Dredd (B. Lemaitre); tub>myc, $y+>$ Gal4 (DE LA COVA et al.); tub>CD2>Gal4 (MORENO AND BASLER). Strain genotypes and sources are listed in the Reagents Table.

Cell competition assays. Competitive and non-competitive control clone assays were performed in wild-type, homozygous mutant or hemizygous mutant backgrounds (DE LA COVA et al. ; DE LA COVA et al. ; MEYER et al. ; ALPAR et al.). Eggs from appropriate crosses were collected on yeasted grape plates for 2-4 hours (or as indicated in figures) and allowed to develop at $25^{\circ} \mathrm{C}$ in a humid chamber for 24 hours. After hatching, larvae were transferred to food vials supplemented with fresh yeast paste at densities of less than 50 larvae per vial to prevent crowding. To measure clonal growth and cell competition in a manner that allows precise temporal control of transgene expression, we used FLP recombinase-mediated intra-molecular recombination (reviewed in (GERMANI et al.)). To generate competitive clones, a tub>myc, $y+>$ Gal4 cassette (where $>$ represents a FLP-recognition target (FRT) site) was used to generate random GFPmarked tub>Gal4 clones (DE LA COVA et al. 2004). Prior to recombination, all cells express the Myc cDNA under control of the tubulin promoter, at a level approximately 1.5 fold over endogenous myc levels (WU AND JOHNSTON 2010). Recognition of the FRT by FLP leads to excision of a myc cDNA and stop sequence and generates cells that heritably express Gal4 (tub>Gal4) and can regulate expression of UAS-genes of interest (e.g., GFP). As the GFP-expressing cells no longer express extra myc, they are subject to competition from surrounding cells that have retained the $>m y c, y+>$ cassette (DE LA CovA et al. 2004). FLP, under heat shock (HS) control, was activated by HS of larvae at $37^{\circ} \mathrm{C}$ for 10 minutes, at 48 hours after egg laying (AEL). Post-HS, larvae were allowed to grow at $25^{\circ} \mathrm{C}$ for 24,48 or 96 hours, as indicated for each experiment. To generate random GFP-marked clones in WT wing discs as noncompetitive controls, the transgenic cassettes act>y+>Gal4 (III chromosome) and tub>CD2>Gal4 (II chromosome) were used as described (DE LA COVA et al. ; DE LA COVA et al. ; MEYER et al. ; ALPAR et al.). In parallel to the competitive cassette, act>Gal4 clones were induced with a $\mathrm{HS}$ at $37^{\circ} \mathrm{C}$ for $6 \mathrm{~min}$, and GFP-positive larvae were selected and dissected at 48 hours after clone induction $(\mathrm{ACl})$. These heat shock times were optimized to generate only few clones per disc, to avoid merged clones. A detailed protocol is available upon request. 
Tissue dissection, fixation and Imaging. Larval carcasses containing mosaic wing discs were dissected and inverted and fixed in 4\% paraformaldehyde in phosphatebuffered saline (PF-PBS) for $20 \mathrm{~min}$ at room temperature and washed with $0.01 \%$ Tween-20 in PBS (PBTw). Hoechst 33258 (Sigma) or DAPI (Invitrogen) was used to stain DNA. Wing discs were mounted in VectaShield Antifade on glass slides. Images were acquired with a Zeiss Axiophot with Apotome, Leica SP5 or Leica LSM710 confocal microscope. Clone area (in square pixels or microns) was measured with Image $\mathrm{J}$ software. Clones were scored in the central area of the wing disc (wing pouch and proximal hinge), where competition is most severe (ALPAR et al.). Clone measurements were log transformed for graphing in Prism. Nonparametric MannWhitney tests or Welch tests were used to determine statistical significance as indicated in each figure.

Immunohistochemistry. Primary antibodies used: guinea pig anti-Grnd (1:200, gift of P. Leopold), rabbit anti-Caspase-3 (1:100, Cell Signaling), and rabbit anti-Dcp-1 (1:100, Cell Signaling) were incubated at $4^{\circ} \mathrm{C}$ overnight with dissected wing discs previously permeabilized with $0.5 \%$ Triton X-100 in PBS (PBTx) for 1 hour and then blocked in PBTw with $5 \%$ normal serum for 1 hour at RT. The samples were incubated with the secondary antibodies Alexa555 and Alexa488 (1:600, Molecular Probes) (pre-absorbed against fixed embryos) for 3 hours at RT in the dark and then washed with PBTw overnight at $4^{\circ} \mathrm{C}$. Hoechst 33258 or DAPI was used to stain DNA. Images were processed and where applicable, assembled from Z-stacks as maximum projections, with Image J software.

RNA in situ hybridization of wing discs. Dissected, fixed and washed larval carcasses were added to $500 \mathrm{ul}$ of $300 \mathrm{mM}$ ammonium acetate and $500 \mathrm{ul}$ of $100 \%$ ethanol and gently mixed, washed in $100 \%$ ethanol for $10 \mathrm{~min}$ at room temperature, washed in xylene/ethanol (1:1) for $10 \mathrm{~min}$, washed in ethanol 3 times, 10 min each, washed in methanol for $2 \mathrm{~min}$, washed in methanol/4\% PF (1:1) for $2 \mathrm{~min}$, fixed again in 4\% PF-PBTw for 10 min, washed in PBTw 5 times for 5 min each, and incubated at RT in hybridization solution/PBTw (1:1) for $15 \mathrm{~min}$; this was replaced by hybridization solution and incubated at the appropriate hybridization temperature for at least $1 \mathrm{hr}$. digRNA probes were added to hybridization solution, mixed well, and denatured at $90^{\circ} \mathrm{C}$ for 
$5 \mathrm{~min}$ followed by incubation on ice for $5 \mathrm{~min}$. The hybridization solution was removed from the carcasses and replaced with the denatured probe in hybridization solution, and incubated at the appropriate temperature overnight. Samples were washed and processed with anti-Digoxygenin antibodies as in Johnston and Edgar (JOHNSTON AND EDGAR). A detailed protocol is available upon request.

Dissociation and FAC sorting of wing disc cell populations. Clonal GFP-marked cell populations were generated in wing discs using the act>y+>Gal4 FRT cassette, UASGFP, and $h s p 70-F l p$ recombinase. FLP expression was induced by HS, allowing expression of Gal4 and activation of desired UAS-transgenes. Clones were generated at the beginning of the third larval instar (72 hr AEL) and conditions optimized to generate mosaic wing discs with approximately $50 \%$ GFP-expressing cells. After induction, clones were allowed to grow for $40 \mathrm{hrs}$, after which wing discs were dissected from larvae in Ringer's solution supplemented with 1\% BSA. Approximately 40-60 wing discs were dissociated in $250 \mu \mathrm{L}$ of Papain $(20 \mathrm{U} / \mathrm{mL})$ and DNase I $(200 \mathrm{U} / \mathrm{mL})$ in Earle's Buffered Salt Solution (all from Worthington Biochemical), at $25^{\circ} \mathrm{C}$ for 40 minutes. GFP-positive and negative cells were sorted and collected using a FACSAria cell sorter (Becton Dickinson). Cells collected in cold PBS were centrifuged at $3,200 \mathrm{~g}$ for $2 \mathrm{~min}$; cell pellets were stored at $-80^{\circ} \mathrm{C}$ and used to isolate total RNA. A detailed protocol is available upon request.

RNA isolation. Total RNA was isolated using TRIZOL from dissociated and FAC-sorted wing disc cells. After adding $160 \mathrm{uL}$ of chloroform, samples were centrifuged at $12,000 \mathrm{~g}$ for 15 minutes at $4^{\circ} \mathrm{C}$. The aqueous phase was transferred to a fresh tube and mixed with $400 \mathrm{ul}$ of isopropyl alcohol. The pellet was washed with $1 \mathrm{ml}$ of $75 \%$ ethanol and centrifuged at $7,500 \mathrm{~g}$ for 5 minutes at $4^{\circ} \mathrm{C}$. The RNA pellet was air-dried, dissolved in RNase-free water and stored at $-80^{\circ} \mathrm{C}$.

Quantitative (real time) RT-PCR. Reverse transcription (RT) to produce singlestranded cDNA was performed using 0.5-1 $\mu \mathrm{g}$ total RNA and SuperScript First-Strand Synthesis kit (Invitrogen). RT reactions containing RT enzyme (RT+) and controls without enzyme (RT-) were performed in parallel. Quantitative PCR (Q-PCR) reactions were performed using LightCycler FastStart DNA MasterPlus SYBR Green I kit (Roche). For each primer set, a standard curve was produced using serially diluted cDNA made 
from WT (Sevelin) wandering stage wing imaginal discs. Reactions were assembled in LightCycler capillaries (Roche) and amplification performed using standard conditions in a Roche LightCycler. In analyzing Q-PCRs, primer appropriate standard curves were used to calculate relative cDNA concentration in each control and experimental sample. Primers to act $5 C$ were used for normalization.

Oligonucleotide Primers. Primers were designed using Primer-Blast with gene sequences (FASTA) obtained from flybase.org. Primers were ordered from MWGBiotech AG. Primer sequences are listed below: act5C F: TGTGACGAAGAAGTTGCTGC, act5C R: AGGTCTCGAACATGATCTGG Traf1 F: CAGCAGATCCAGATGCTCAA, Traf1 R: TCTTGCTGGGATCCACTTTC egr F: CATTTCCACTTGAGCAGCAG, egr R: GCACGATTCCGATTGAGATT

\section{Results}

\section{egr expression is upregulated in WT loser cells and required for their elimination.}

In genome-wide gene-chip expression studies that aimed to identify genes expressed by competing wing disc cell populations (de la Cova ms in prep), we found that egr and Traf4 transcripts were part of a cluster of mRNAs upregulated in WT cells that grew near GFP-marked, Myc-expressing cell clones (WT loser cells). We were intrigued by this finding since Egr and Traf4 function in activation of the JNK pathway, which has been observed in this context of cell competition but whose contribution to the loser fate has remained uncertain (DE LA COVA et al. ; MORENO AND BASLER). egr and Traf4 are both expressed in wing discs (AYYAR et al. 2007; BROWN et al. ; SANCHEZ et al.). However, ModEncode RNA-sequencing data and proteomic studies indicate that expression of both genes is quite low in third instar larval wing discs (BROWN et al. ; CASAS-VILA et al.), and consistent with this we were unable to detect the increase in egr or Traf4 expression in loser cells with immunocytochemistry or by RNA in situ hybridization (except in the adult muscle precursor cells, which express egr at high levels; data not shown). To validate our transcriptomic results, we isolated WT loser cells and Myc expressing winner cells from wing discs in which Myc-mediated cell competition was induced using fluorescent activated cell sorting (FACS); egr and Traf4 mRNA expression was then measured by quantitative RT-PCR. These experiments determined that egr mRNA was modestly up-regulated, approximately 1.9-fold, relative to WT cells sorted from a GFPonly control, and expression of Traf4 mRNA was increased approximately 1.3-fold (Fig. 
1A). Up-regulation of egr and Traf4 expression was specific to WT losers, as the level of their transcripts was unchanged in GFP-marked, Myc-expressing winner cells sorted from the same wing discs (Fig. 1A).

Over-expression of egr under Gal4 control induces cell death in the developing eye and wing (IGAKI et al. 2002; MORENO et al. 2002b). Accordingly, cell death was strongly induced when egr expression was driven by Gal4 in GFP-marked cell clones in wing discs (Fig. 1B-C). Cells within the wing pouch were particularly sensitive to its effects, such that many of the egr-expressing clones were eliminated from this region after only 24 hours. Many dying cells, visualized using an antibody specific to cleaved and activated Caspase-3 (Cas-3), were visible in a basal focal plane under the wing pouch (Fig. 1C').

The finding that egr mRNA is specifically increased in the loser cells in Myc-induced cell competition suggested Egr could have a role in their apoptotic elimination. Competitive elimination of loser cells can be quantified in an assay that measures the competitive effects of Myc against WT clonal cell populations generated in a growing tissue such as the wing disc (DE LA COVA et al.). The basis of the assay is a transgenic FRT cassette, tub>myc, $y+>$ Gal4 (> denotes FRT; Fig. 1D-E). When intact, the transgene drives myc mRNA expression under the control of the tubulin promoter at a level approximately $50 \%$ higher than the level of endogenous myc, but transcription is terminated before the Gal4 coding sequences (WU AND JOHNSTON). Upon controlled expression of Flp recombinase, the $>m y c, y+$ sequences are stochastically excised, allowing heritable expression of Gal4 (tub>ga/4) and activation of UAS-GFP. Cells in the tub>gal4 clones are essentially WT, yet are at a competitive disadvantage due to the non-clonal surrounding cells that continue to express elevated Myc from the intact $t u b>m y c, y+>g a l 4$ transgene. A consequence of this disadvantage is that in larval stages, the "loser" cells in tub>Gal4 clones die at increased frequency due to induction of the hid pro-apoptotic gene. The loser clones are thus significantly smaller (Fig.1F and G, WT Losers) than non-competitive control clones that are generated in parallel under non-competitive conditions (e.g., Fig.1F and G, WT Ctls; see Methods for details). The growth disadvantage of the loser clones is completely rescued by genetic loss of hid (DE LA COVA et al.), indicating that the small size of loser clones is a direct result of increased cell death (DE LA COVA et al. 2004; MORENO AND BASLER ; DE LA COVA et al. 2014; MEYER et al. 2014; MERINO et al.).

We tested whether the egr ${ }^{3 A G}$ null allele (KODRA et al.) altered the fate of the loser 
cells in this competition assay. In a WT background of cells expressing Myc, generation of GFP-marked loser clones were competitively eliminated from the wing disc, leading to a significant reduction in clone size compared to non-competitive control clones generated in parallel (Fig. 1D-F). However, in the egr ${ }^{3 A G}$ background, competition was abolished between the Myc expressing cells and the clones of WT loser cells (Fig. 1F, egr ${ }^{3 A G}$ ). Indeed, clones generated in both competitive and non-competitive contexts grew at the same rate and to the same final size (Fig. $1 \mathrm{~F}$, egr $\left.^{3 A G}\right)$. To determine whether the requirement for egr was specific to the "loser" cells we expressed RNAi directed against egr (UAS-egr $r^{\prime R}$ ) specifically in the clonal cell populations. The treatment did not affect the growth or survival of cells in controls, but its expression in loser cells completely prevented their competitive elimination (Fig. 1G). These results indicate that the upregulation of egr expression in WT loser cells, which is a consequence of their competitive interactions with Myc expressing cells, leads to a specific requirement for Egr for their elimination from the wing disc.

\section{Grnd/TNFR is required to eliminate WT loser cells from wing discs}

The Drosophila genome encodes two TNFRs, Wgn and Grnd. In wing discs, wgn mRNA expression is restricted to the wing pouch (Fig. 2A), with an expression pattern somewhat complementary to that of egr, in that it is highest in the wing pouch and lowest in the notum. Previous reports of egr over-expression in the eye disc linked Egr activity to wgn, the adaptors Traf4 and Traf6, and the JNK signaling pathway (IGAKI et al. 2002; KANDA et al. 2002; GEUKING et al. 2005). Furthermore, the highest wgn expression corresponds to the region of the disc subject to the most severe effects of cell competition (ALPAR et al. 2018). As wgn is located on the $\mathrm{X}$ chromosome, we examined clone growth and cell competition in wing discs from WT and $w^{22} n^{22}$ (RUAN et al.) male larvae. As expected, after a 48hr growth period, loser clones in WT wing discs were significantly smaller than non-competitive control clones (Fig. 2B, WT Ctls vs WT Losers), and loser clones in $w g n^{22}$ discs were similarly small (Fig. 2B, wg $n^{22}$ Losers). However, the non-competitive control clones in $w g n^{22}$ discs also grew poorly, growing to much smaller sizes than control clones in WT wing discs (Fig. 2B, wg ${ }^{22} \mathrm{Ctls}$ ). The small clone size was not due to smaller overall disc size, as all the discs were similar in size (Fig. 2B'). The similar size of both control clones and loser clones in $w^{2} n^{22}$ discs make it difficult to make conclusions regarding the role of Wgn in cell competition. Given that loser clones are measured relative to control clones grown in the same genetic 
background, one interpretation of these results is that although all clonal growth is reduced relative to $\mathrm{WT}$, in $w^{2} n^{22}$ mutants the competitive elimination of loser cells is suppressed, allowing those loser clones to grow as well as their cognate noncompetitive controls.

In contrast to Wgn, the TNFR Grnd is uniformly expressed in wing discs and is present on the sub-apical membrane of the cells (Fig. 2C, C') (ANDERSEN et al. 2015). Complete loss of grnd does not affect viability, and like Wgn, Grnd binds to and requires Traf6 for signaling (ANDERSEN et al. 2015). The expression of Grnd protein in loser clones as well as control clones in the wing pouch of third instar larval wing discs appeared to be localized normally at the apical surface of cells (Fig. 2C'). To test for a requirement for Grnd in cell competition, we used the trans-heterozygous combination grnd $^{\mathrm{Df}} /$ grnd $^{\mathrm{Minos}}$, which expresses no detectable Grnd protein (ANDERSEN et al. 2015). The absence of grnd suppressed much of the elimination of the loser cells, allowing loser clones to grow to sizes similar to the controls (Fig. 2D, grnd ${ }^{D f / M i}$ Ctls vs Losers). In contrast to loss of wgn, grnd loss had no effect on the size of non-competitive control clones (Fig. 2D, grnd ${ }^{D / M i}$ Ctls vs WT Ctls). This indicates that the role of Grnd in the loser clones was specific to the competitive context. To determine whether Grnd was required within the loser cells, we expressed UAS-grnd-RNAi ( $\left.g r n d^{\prime R}\right)$ in the loser cells. Again, knock-down of grnd impaired cell competition, but had no effect on cell clones in a noncompetitive environment (see Fig. 5G). Together, our results suggest that once induced in the loser cells during cell competition, Egr functions with Grnd to promote their elimination. The role of Wgn remains ambiguous, but as both Wgn and Grnd are able to bind Egr, it is possible that they function cooperatively.

Grnd binds Egr via its extracellular N-terminal CRD domain, which results in signaling transmitted through its intracellular C-terminal domain (ANDERSEN et al. 2015). However, expression of only the intracellular portion of Grnd, UAS-grnd ${ }^{\text {intra }}$, is sufficient to activate JNK signaling and apoptosis in an Egr-independent manner (ANDERSEN et al. 2015). Apoptosis induced by expression of UAS-grnd ${ }^{\text {intra }}$ is efficiently suppressed in the hep ${ }^{r 75}$ mutant background (ANDERSEN et al. 2015), confirming that Grnd functions upstream of the JNK signaling cascade. To determine whether intracellular signaling through Grnd is required to eliminate loser cells in cell competition we expressed UASgrnd $^{\text {lintra }}$ in wing discs. As a control, we expressed UAS-grnd ${ }^{\text {extra }}$, a cognate transgene containing only the extracellular portion of Grnd, which binds to Egr but does not activate JNK signaling (ANDERSEN et al. 2015). Expression of UAS-grnd ${ }^{\text {extra }}$ using $r n G a l 4$, which 
drives expression in the wing pouch, led to only a slight, patchy rise in cell death (Fig. 3A, A'), consistent with previous results (ANDERSEN et al. 2015). Similarly, grnd ${ }^{\text {extra }}$ had no effect on their growth when expressed in clones, in either a non-competitive or a competitive environment (Fig. 3B, C). However, rn-Gal4-directed expression of grndintra led to massive cell death, substantially reducing the size of wing discs (Fig. 3D, D'). When we expressed $g r n d^{\text {intra }}$ in GFP-marked cell clones, despite being detectable initially (Fig. 3E-F insets), wing discs were completely devoid of GFP-positive cells by $48 \mathrm{hr} \mathrm{ACl}$, indicating that all of the clones were eliminated (Fig. 3E-F). Thus, our results confirm that expression of grnd $^{\text {intra }}$, and by inference Grnd activation, transmits a powerful deathinducing signal within cells (ANDERSEN et al. 2015). Moreover, complete elimination from the wing epithelium was the outcome of $g r n d^{\text {intra }}$-expressing clones in both noncompetitive (Fig. 3E) and competitive environments (Fig. 3F).

\section{Traf4 and Traf6 contribute to elimination of wild-type loser cells}

In Drosophila, intracellular signaling via TNFRs is transduced through the TNF receptor-associated factors Traf4 (formerly known as dTraf1) and Traf6 (formerly dTraf2). TNF-TNFR ligation has been shown to activate both the JNK and the NF-kB signaling pathways, possibly because different intracellular signals can be transduced by different Traf adaptors (CHA et al. 2003). Traf4 physically associates with Wgn and Grnd, as well as Tak1, Tab2 and the Sterile-20 kinase, Misshapen (LIU et al. 1999; CHA et al. 2003; ANDERSEN et al. 2015). Expression of Traf4 is sufficient to induce JNK activity and cell death (CHA et al. 2003). Although signaling mediated by Traf4 is directed primarily through the JNK pathway, it has also been implicated in NF-KB -mediated responses (SHEN et al. 2001; AYYAR et al. 2007). Moreover, in wing discs, Traf4 is required to activate the NF-KB Relish for maintenance of proneural gene expression (AYYAR et al. 2007). Since Traf4 mRNA was modestly but specifically upregulated in loser cells (Fig. $1 \mathrm{~A})$, we tested for its requirement by eliminating Traf4 function during cell competition. Competition was suppressed when UAS-Traf4 ${ }^{I R}$, encoding a Traf4 inverted repeat (IGAKI et al.), was expressed specifically in the loser cells (Fig. 3G, Losers), whereas its expression did not affect the growth of control clones (Fig. 3G, Ctls). The elimination of loser cells was also suppressed in larvae carrying the severe Traf4 ${ }^{e x 1}$ allele (CHA et al. 2003), and resulted in loser clones that were comparable in size to control clones in a non-competitive environment (Fig. 3H). 
The Traf6 adaptor also associates with the intracellular domains of Wgn and Grnd (ANDERSEN et al. 2015). Unlike Traf4, ectopic expression of Traf6 is not sufficient to activate JNK signaling nor induce apoptosis (CHA et al. 2003; GEUKING et al. 2005), but it can participate in both JNK and NF-kB signaling. Traf6 functionally interacts with Pelle, the Toll pathway kinase, and in S2 cells Pelle and Traf6 cooperate in the activation of the NF-KB dorsal (SHEN et al. 2001). To examine the role of Traf6 in cell competition, we expressed the RNAi transgene UAS-Traf6 ${ }^{I R}$ (IGAKI et al.) in non-competitive control and competitive loser clones. Like loss of Traf4, knock-down of Traf6 in the loser cells suppressed their elimination, allowing the clones to grow to the same size as noncompetitive control clones (Fig. 3I). The loss of Traf6, like that of Traf4, had no obvious effect on cells in non-competitive control clones, indicating that its role was specific to the loser cells in the competitive context. Thus, the elimination of loser cells requires the function of both Traf4 and Traf6.

Collectively, our results suggest that downstream of Egr and Grnd, Traf4 and Traf6 both participate in transducing the signal within the loser cells that leads to their death. The two adaptors could function together in heterotypic complexes in the same signaling pathway. However, given the lack of redundancy between these adaptors in the WT loser cells, they could function in parallel pathways during competitive signaling: for example, Traf4 might promote JNK signaling, while Traf6 functions in the CCSM in activation of Relish.

\section{JNK signaling has a minor role in the elimination of WT loser cells.}

Transduction of JNK signaling downstream of Egr/Grnd activity has a critical requirement for the MAP3K TGF-beta activated kinase Tak1 (ANDERSEN et al. 2015). In previous studies, Tak1 was found to be dispensable for the elimination of loser cells in Myc- and in $R p^{-/ t}$ - mediated cell competition (MEYER et al. 2014). However, given our finding that Egr is upregulated in WT loser cells, we tested for a role of Tak1 using two different Tak1 alleles that harbor point mutations in the kinase domain and are considered nulls (VIDAL et al. 2001; DELANEY et al. 2006). Hemizygous Tak1²/Y or Tak $1^{1} / Y$ larvae are viable, allowing us to study their function in wing discs from male larvae. We induced cell competition in $\operatorname{Tak}^{2} / Y$ or in $T a k 1^{1} / Y$ mutant larvae and examined the clones in wing discs, and found that neither mutation was sufficient to suppress loser cell elimination (Fig. 4A). Indeed, loser clones in wing discs from both Tak1 mutants grew as poorly as loser clones in WT wing discs (Fig. 4A). This result 
confirms that the absence of Tak1 is unnecessary for the elimination of loser cells in this competitive context. It nevertheless raises an interesting conundrum, since Tak1 is on the one hand required downstream of TNFR to activate JNK signaling (VIDAL et al. ; DELANEY et al. ; STRONACH et al. ; ANDERSEN et al.), and on the other, it plays crucial roles in the activation of Rel in the immune response to pathogenic infection (SILVERMAN et al. ; PARK et al.).

Ectopic expression of egr in a stripe bisecting the wing disc induces massive cell death, measured by immunostaining with an antibody against active Caspase-3 (Cas-3) (Fig. 4B). This death is mediated by Egr's activation of the JNK pathway, as it is largely suppressed in $h e p^{r 75} / Y$ larvae. hep ${ }^{r 75}$ is a severe loss of function allele of the Hep/MKK7 protein, required to transmit JNK signaling (GLISE et al.) (Fig. 4C). However, only a fraction of the cell death in loser clones was suppressed in hep ${ }^{175}$ mutants (DE LA COVA et al. 2004), suggesting that JNK pathway activity is not sufficient to account for their elimination. Indeed, in WT loser clones generated under competitive conditions, $45 \%$ of the clones contained Cas-3 positive cells at 24hr ACl (Fig. 4D, D', F). Likewise, at the same time point, $42 \%$ of loser clones generated in a hep $p^{r 75}$ mutant background contained Cas-3 positive cells, although on average they contained fewer Cas-3 positive cells/clone (Fig. 4E, E', F), suggesting that loss of hep reduced, but did not prevent loser cell death. Consistent with this, hep ${ }^{\text {r75 }}$ loser cells were ultimately eliminated from the wing discs as effectively as WT loser cells. By $50 \mathrm{hr} \mathrm{ACl,} \mathrm{the} \mathrm{median} \mathrm{size} \mathrm{of} \mathrm{loser} \mathrm{clones}$ in hep ${ }^{r 75}$ mutant males was similar to that of loser clones in a WT background, and both were significantly smaller than non-competitive control clones (Fig. 4G). To further test the requirement for the JNK pathway, we expressed a dominant negative form of the Jun kinase Basket (Bsk), which functions downstream of Hep (RIESGO-ESCOVAR et al. ; SLUSS et al.), in the loser clones. Expression of UAS-Bsk ${ }^{D N}$ in the loser cells allowed the clones to grow slightly larger that WT loser clones, again implying that cell death was partially suppressed (Fig. 4H). Overall, however, the clones were still significantly smaller than controls, indicating that competition still occurred.

Thus, loss of any of three essential intermediaries of the JNK pathway can slightly diminish loser cell apoptosis, but is not sufficient to prevent their ultimate elimination from the wing disc. Altogether, our results indicate that canonical JNK activity plays a minor role in the death and elimination of WT loser cells.

\section{Egr/Grnd function in WT Loser cells is independent of JNK activity}


Together, our results establish that egr expression is induced in loser cells during cell competition, and that its function is required for the cells' elimination from the wing disc epithelium. Our experiments also indicate that transduction of the Egr signal requires Grnd, but notTak1, hep or bsk, each of which are critical for JNK signaling and commonly activated downstream of Egr/Grnd signaling. Indeed, downstream of Traf4 and Traf6, our data indicate that canonical JNK activity is not required for cell competition. Implicit in these conclusions is that, in the loser cells, Egr function is largely independent of JNK activity. Since the NF-KB Rel regulates expression of Hid and apoptosis in loser cells (MEYER et al. 2014; NAGATA et al. 2019), one explanation for our results is that in the loser cells, signaling from Egr/Grnd participates with or reinforces the CCSM in the activation of Rel. We reasoned that if this was the case, in the competitive context, activation of Egr/Grnd signaling in a Tak1 mutant should not suppress loser cell death (i.e., the cells would still be eliminated). Conversely, in a noncompetitive context, loss of Tak1 would be predicted to completely block the canonical JNK signaling downstream of Egr/Grnd activation (Fig. 5B) and allow the cells to survive.

We tested these ideas by expressing grnd ${ }^{\text {intra }}$ in cell clones in $T a k 1^{2} / Y$ hemizygous male larvae, and quantifying the clones in wing discs from both non-competitive control and competitive contexts. Female $T a k 1^{2} /+$ larvae, which clonally expressed $g r n d^{\text {intra }}$ but were heterozygous for Tak1, served as an internal control and exhibited massive death induced by grnd ${ }^{\text {intra }}$ expression that severely affected larval growth (Fig. 5A) (ANDERSEN et al. 2015). By contrast, hemizygous $T a k 1^{2} / Y$ males from the same cross were unable to transduce the grnd $^{\text {intra }}$-mediated death signal, allowing them to grow normally (Fig. $5 A)$. Induction of cell competition in WT male larvae shows the normal growth disadvantage in loser cells compared to non-competitive controls (Fig. 5B), whereas expression of grnd $^{\text {intra }}$ in either control or loser clones completely prevented their growth due to acute cell death (Fig. 3D-F and represented in 5C as 'no clones'). As predicted, in a non-competitive context, the absence of Tak1 completely prevented grnd ${ }^{\text {intra-induced }}$ cell death, allowing clones to grow to the same size as non-expressing WT controls (Ctls, Fig. 5D vs. 5B). In the competitive context, preventing JNK activity with loss of Tak1 also allowed the growth of clones (Losers, Fig. 5D). However, Tak1 loss did not prevent the cells from acquiring the "loser" fate: these clones were still significantly smaller than non-competitive control clones (Losers, Fig. 5D vs 5B). This result is consistent with the lack of a requirement for Tak1 in cell competition (MEYER et al.), and indicates that in the loser cells, the absence of Tak1 selectively prevented JNK- 
dependent cell death driven by $g r n d^{\text {intra }}$, whereas their death induced through the CCSM still occurred.

\section{Dredd and Grnd have different genetic relationships in non-competitive versus competitive environments}

The Drosophila caspase-8 ortholog Dredd functions in the CCSM and is required upstream of Rel for loser cell death (MEYER et al. 2014). Dredd is also required for immune signaling by the IMD pathway, and its over expression is sufficient to induce proteolytic activation of Rel and leads to Rel-dependent gene expression in immune competent tissues (STOVEN et al. 2003; ZHOU et al. 2005). In wing discs, ectopic expression of Dredd leads to Rel-dependent cell death (MEYER et al. 2014). In a competitive context, the death of WT loser cells is dominantly suppressed in Dredd/+ $R e l /+$ double heterozygous mutants, suggesting that the loser cells have increased sensitivity to Dredd activity (MEYER et al. 2014). Notably, however, Dredd is not required for cell death induced by canonical JNK signaling downstream of Egr (IGAKI et al. 2002; MORENO et al. 2002b).

Thus, to further explore the relationship between the JNK pathway and the CCSM, we tested for genetic interactions between JNK signaling and Dredd. Expression of UAS-Dredd in cell clones, even under under non-competitive conditions, leads to Reldependent apoptosis (MEYER et al.) and significantly reduces clone size compared to non-expressing controls (Fig. 5E-F, Ctls); whether JNK signaling plays a role in this process is unknown. In the competitive context, where Dredd is already active as part of the CCSM in loser cells, expression of UAS-Dredd enhances their elimination (Fig. 5E-F, Losers) (MEYER et al.). Furthermore, RNAi-mediated knockdown of grnd, like its complete loss (Fig. 2E), fully suppresses the competitive elimination of loser cells (Fig. $5 \mathrm{G})$. Given that loss of grnd prevents Egr-induced activation of JNK signaling (ANDERSEN et al. 2015), we tested whether Dredd-induced cell killing required input from Egr/Grnd activity. Based on our findings, we postulated that if signaling through Grnd was blocked with grnd-RNAi, the cell death induced by expression of UAS-Dredd would rely solely on the CCSM. Indeed, in the non-competitive context, grnd knock-down was unable to suppress the clone size reduction caused by Dredd expression (Fig. 5F, $5 \mathrm{H}$, Ctls), indicating that Dredd-induced cell death does not require input from Grnd. By contrast, in the competitive context, loser cells that expressed UAS-grnd ${ }^{\prime R}$ and UASDredd were still efficiently eliminated (Fig. 5H Losers). Thus, under both non-competitive 
and competitive conditions, Dredd activity is epistatic to Grnd and does not require JNK signaling to induce cell death.

Together, these experiments argue that the induction of egr expression in the loser cells and its subsequent autocrine signaling via Grnd reinforces the CCSM-mediated cell death response that limits loser clone growth. However, they also argue that Egr/Grnd can activate two parallel signaling pathways in loser cells: one that is Tak1 independent and steers signaling to the CCSM, in which cell death is mediated by Dredd and Rel (MEYER et al.); and another that directs signaling through the canonical JNK pathway mediators (DE LA COVA et al. ; MORENO AND BASLER), but provides only a minor contribution to the death of the loser cells. We note that the enhancement of loser cell elimination conveyed by Dredd expression was slightly diminished in the absence of grnd (Fig. 5H, 5F Losers), which could be interpreted as evidence of parallel, additive signaling. Our results thus imply that the JNK and CCSM signaling pathways function in parallel during cell competition, but that the CCSM predominates in the killing of the loser cells. Our results make it clear that complex signaling inputs are deployed in loser cells, and underscore that WT loser cells are subject to qualitatively different survival and death cues than are genotypically-identical WT cells in a non-competitive environment.

\section{Discussion}

Although the JNK pathway is known to be activated in Myc-induced cell competition, the relative contribution of this pathway to the elimination of the WT loser cells, and how JNK signaling and the CCSM interact, have remained unclear. We have attempted to understand these interactions with a series of genetic experiments. We demonstrate that competition between Myc expressing cells and WT cells leads to a 2-fold increase in expression of the TNF Egr specifically in the loser cells. We establish that loss of Egr/TNF or Grnd/TNFR suppresses the elimination of loser cells, indicating that each are functionally required in the process. Although Egr is known to kill cells via JNK activation (IGAKI et al. 2002; MORENO et al. 2002b), we find it notable that in competition assays, loss of JNK signaling only partially blocks the death of the loser cells (Fig. 4E-H) (DE LA COVA et al. 2004). Our results argue that in Myc-induced cell competition, signaling by Egr/Grnd in loser cells bifurcates upstream of Tak1, to facilitate and/or potentiate the activity of the CCSM in loser cells in a JNK pathway-independent manner. Collectively, our experiments reinforce earlier findings that despite its activation in cell 
competition JNK signaling is not sufficient for the elimination of the loser cells (DE LA COVA et al. 2004), in contrast to Rel, which is critical for loser cell death (MEYER et al. 2014; NAGATA et al. 2019).

Our experiments suggest that during Myc-induced cell competition, the CCSM, composed of factors from the IMD and Toll pathways that signal to induce the death of WT loser cells and thereby limit their growth in the wing disc (MEYER et al. 2014; ALPAR et al. 2018), induces expression of a loser-specific program of gene expression that includes egr and Traf4, as well as hid (MEYER et al. 2014; NAGATA et al. 2019). Our results suggest a mechanism for how Egr functions in loser cell death. We propose that competitive signaling leads to induction of egr expression and production of Egr protein in the loser cells, whereby Egr then signals in an autocrine manner via Grnd. We speculate that activation of Egr/Grnd signaling then transmits information within the loser cells in two ways. First, and predominantly, it reinforces CCSM signaling, possibly by potentiating Dredd and Rel activity and intensifying apoptosis. Second, via Tak1, Egr/Grnd activates canonical JNK signaling, which provides a minor contribution to the death of the cells.

Although the exact mechanism by which the Egr/Grnd signal branches from JNK to the CCSM occurs remains to be determined, our data suggest that it occurs at the level of the Traf adaptors. Both Traf4 and Traf6 are required in our assays for loss of the loser cells, whereas the absence of Tak1, does not affect the outcome of cell competition even while it abrogates JNK signaling. Our genetic interaction tests with Grnd and Dredd suggest that downstream of Egr/Grnd activation, Traf4/6 facilitate and/or enhance Dredd activity, which is already functional in loser cells (MEYER et al.), thereby augmenting Rel-regulated, loser-specific gene expression and cell death.

What is the nature of the interactions between the activities of the JNK pathway and the CCSM? One possibility takes into account findings that JNK activation during the immune response is short-lived (SLUSS et al. 1996), in part because of feedback from Rel that dampens JNK signaling by promoting the degradation of Tak1 (PARK et al. 2004; ZHOU et al. 2005). Alternatively, Traf4 is known to activate the dorsal (dl)/NF-kB pathway (LIU et al. 1999; SHEN et al. 2001), and the egr locus is a target of $\mathrm{dl}$ in early embryos (MARKSTEIN et al. 2002; STATHOPOULOS et al. 2002). Egr/Grnd signaling could thus lead to dl/Dif activity as well as Rel, strengthening the outcome in the loser cells. This could potentially take the form of a feed-forward activity that stabilizes egr expression in some loser cells, although the level of Egr in a loser cell would appear to 
be quite modest, since we have not been able to detect it in situ. Clonal assays make it clear that the elimination of loser cells is stochastic, with some loser cells falling to the death-inducing signals easier than others. The probability of loser cell death could be influenced by several independent factors, including accumulation of the proapoptotic factor Hid, which in turn would be influenced by inputs to the hid locus via the activities of the CCSM and JNK signaling.

Egr can be produced as a membrane bound protein or in a soluble form, after proteolytic processing (KAUPPILA et al. 2003; NARASIMAMURTHY et al. 2009). Although we did not address which form is made by and/or acts on the loser cells, we did not detect evidence of non-cell autonomous killing activity. Since Grnd is ubiquitously expressed in wing discs, expression of Egr in loser cells could feasibly affect the winner cells as well. The alternate TNFR, Wgn, is necessity for elimination of polarity deficient cells that compete with WT cells in the eye disc (IGAKI et al.), but in our experiments, the reduced growth of the control clones in $w g n^{22}$ mutants makes an assessment of its role cell competition difficult. However, given its expression pattern (Fig. 2A), it is possible that in the Myc-induced competition paradigm, Wgn and Grnd act semi-redundantly to promote elimination of loser cells in the wing pouch, where cells are most severely subject to competitive interactions (ALPAR et al.). This will be an interesting idea to explore in future work.

We note that the intersection of the CCSM and JNK signaling that we describe here may be unique to the context of Myc-induced competition (and possibly $R p /+$, where both pathways have also been documented (MORENO et al. ; TYLER et al. ; MEYER et al.)). Competition between the polarity deficient scrib mutant cells and WT cells also utilizes Egr-JNK signaling to eliminate the scrib loser cells, but this is Tak1-dependent, and as mentioned, also requires Wgn (IGAKI et al. 2006). Future studies will be important to sort out these differences. Regardless, our results help to explain the basis of the relative contributions of the CCSM and JNK activity to Myc-induced cell competition. In addition, they add to the growing body of work that illustrates the remarkable signaling flexibility that occurs between cells in competitive contexts, allowing cells to tune their response to a given cellular or environmental context.

ACKNOWLEDGEMENTS. We thank P. Leopold, B. Lemaitre, M. Miura. P. Barker and T. Igaki for Drosophila strains, P. Leopold for antibodies, T. Jessell for the generous use of a FACs-Arias and I. Schlieren for guidance and assistance with cell sorting. We are 
grateful to members of the Johnston lab for helpful advice, C. Cary, P. Guevarra and D. Miranda for excellent technical support, and L. Alpar for drawings. We are indebted to the BDSC (supported by NIH P40OD018537), DGRC (supported by NIH 2P40OD010949) and FlyBase (ATTRILL et al.) for their valuable resources. Funding for this work was provided by grants from the NIH (R01GM078464 and R35GM131871) and the $\mathrm{NCl}$ (R01CA192838) to L.A.J.

AUTHOR CONTRIBUTIONS. All authors conceived and designed experiments. A.K., C. de la C., A.S.S. conducted experiments. A.K., C.de la C., A.S.S. and L.A.J. analyzed results. All authors contributed to writing the paper. L.A.J. supervised the project and provided grant support.

\section{REFERENCES}

Alpar, L., C. Bergantinos and L. A. Johnston, 2018 Spatially Restricted Regulation of Spatzle/Toll Signaling during Cell Competition. Dev Cell 46: 706-719 e705.

Andersen, D. S., J. Colombani, V. Palmerini, K. Chakrabandhu, E. Boone et al., 2015 The Drosophila TNF receptor Grindelwald couples loss of cell polarity and neoplastic growth. Nature 522: 482-486.

Attrill, H., K. Falls, J. L. Goodman, G. H. Millburn, G. Antonazzo et al., 2016 FlyBase: establishing a Gene Group resource for Drosophila melanogaster. Nucleic Acids Res 44: D786-792.

Ayyar, S., D. Pistillo, M. Calleja, A. Brookfield, K. Gittins et al., 2007 NF-kappaB/Relmediated regulation of the neural fate in Drosophila. PLoS One 2: e1178.

Banreti, A. R., and P. Meier, 2020 The NMDA receptor regulates competition of epithelial cells in the Drosophila wing. Nat Commun 11: 2228.

Brown, J. B., N. Boley, R. Eisman, G. E. May, M. H. Stoiber et al., 2014 Diversity and dynamics of the Drosophila transcriptome. Nature 512: 393-399.

Casas-Vila, N., A. Bluhm, S. Sayols, N. Dinges, M. Dejung et al., 2017 The developmental proteome of Drosophila melanogaster. Genome Res 27: 1273-1285.

Cha, G. H., K. S. Cho, J. H. Lee, M. Kim, E. Kim et al., 2003 Discrete functions of TRAF1 and TRAF2 in Drosophila melanogaster mediated by c-Jun N-terminal kinase and NF-kappaB-dependent signaling pathways. Mol Cell Biol 23: 7982-7991.

Chinchore, Y., G. F. Gerber and P. J. Dolph, 2012 Alternative pathway of cell death in Drosophila mediated by NF-kappaB transcription factor Relish. Proc Natl Acad Sci U S A 109: E605-612.

de la Cova, C., M. Abril, P. Bellosta, P. Gallant and L. A. Johnston, 2004 Drosophila myc regulates organ size by inducing cell competition. Cell 117: 107-116.

de la Cova, C., N. Senoo-Matsuda, M. Ziosi, D. C. Wu, P. Bellosta et al., 2014 Supercompetitor status of Drosophila Myc cells requires p53 as a fitness sensor to reprogram metabolism and promote viability. Cell Metab 19: 470-483.

Delaney, J. R., S. Stoven, H. Uvell, K. V. Anderson, Y. Engstrom et al., 2006 Cooperative control of Drosophila immune responses by the JNK and NF-kappaB signaling pathways. EMBO J 25: 3068-3077. 
Erturk-Hasdemir, D., M. Broemer, F. Leulier, W. S. Lane, N. Paquette et al., 2009 Two roles for the Drosophila IKK complex in the activation of Relish and the induction of antimicrobial peptide genes. Proc Natl Acad Sci U S A 106: 9779-9784.

Germani, F., C. Bergantinos and L. A. Johnston, 2018 Mosaic Analysis in Drosophila. Genetics 208: 473-490.

Geuking, P., R. Narasimamurthy and K. Basler, 2005 A genetic screen targeting the tumor necrosis factor/Eiger signaling pathway: identification of Drosophila TAB2 as a functionally conserved component. Genetics 171: 1683-1694.

Glise, B., H. Bourbon and S. Noselli, 1995 hemipterous encodes a novel Drosophila MAP kinase kinase, required for epithelial cell sheet movement. Cell 83: 451-461.

Hof-Michel, S., L. Cigoja, S. Huhn and C. Boekel, 2020 Innate immune signalling drives loser cell elimination during stem cell competition in the Drosophila testis. bioRxiv

Hu, S., and X. Yang, 2000 dFADD, a novel death domain-containing adapter protein for the Drosophila caspase DREDD. J Biol Chem 275: 30761-30764.

Igaki, T., H. Kanda, Y. Yamamoto-Goto, H. Kanuka, E. Kuranaga et al., 2002 Eiger, a TNF superfamily ligand that triggers the Drosophila JNK pathway. EMBO J 21:30093018.

Igaki, T., R. A. Pagliarini and T. Xu, 2006 Loss of cell polarity drives tumor growth and invasion through JNK activation in Drosophila. Curr Biol 16: 1139-1146.

Igaki, T., J. C. Pastor-Pareja, H. Aonuma, M. Miura and T. Xu, 2009 Intrinsic tumor suppression and epithelial maintenance by endocytic activation of Eiger/TNF signaling in Drosophila. Dev Cell 16: 458-465.

Johnston, L. A., 2009 Competitive interactions between cells: death, growth, and geography. Science 324: 1679-1682.

Johnston, L. A., and B. A. Edgar, 1998 Wingless and Notch regulate cell-cycle arrest in the developing Drosophila wing. Nature 394: 82-84.

Kanda, H., T. Igaki, H. Kanuka, T. Yagi and M. Miura, 2002 Wengen, a member of the Drosophila tumor necrosis factor receptor superfamily, is required for Eiger signaling. J Biol Chem 277: 28372-28375.

Kauppila, S., W. S. Maaty, P. Chen, R. S. Tomar, M. T. Eby et al., 2003 Eiger and its receptor, Wengen, comprise a TNF-like system in Drosophila. Oncogene 22: 4860-4867.

Kim, C. H., D. Paik, F. Rus and N. Silverman, 2014 The caspase-8 homolog Dredd cleaves Imd and Relish but is not inhibited by p35. J Biol Chem 289: 20092-20101.

Kleino, A., and N. Silverman, 2014 The Drosophila IMD pathway in the activation of the humoral immune response. Dev Comp Immunol 42: 25-35.

Kodra, A., C. de la Cova, A. R. Gerhold and L. A. Johnston, 2020 Widely used mutants of eiger, encoding the Drosophila Tumor Necrosis factor, carry additional mutations in the NimrodC1 phagocytosis receptor. G3.

Liu, H., Y. C. Su, E. Becker, J. Treisman and E. Y. Skolnik, 1999 A Drosophila TNFreceptor-associated factor (TRAF) binds the ste20 kinase Misshapen and activates Jun kinase. Curr Biol 9: 101-104.

Markstein, M., P. Markstein, V. Markstein and M. S. Levine, 2002 Genome-wide analysis of clustered Dorsal binding sites identifies putative target genes in the Drosophila embryo. Proc Natl Acad Sci U S A 99: 763-768.

Merino, M. M., C. Rhiner, J. M. Lopez-Gay, D. Buechel, B. Hauert et al., 2015 Elimination of unfit cells maintains tissue health and prolongs lifespan. Cell 160: 461-476.

Meyer, S. N., M. Amoyel, C. Bergantinos, C. de la Cova, C. Schertel et al., 2014 An ancient defense system eliminates unfit cells from developing tissues during cell competition. Science 346: 1258236. 
Moreno, E., and K. Basler, 2004 dMyc transforms cells into super-competitors. Cell 117: 117-129.

Moreno, E., K. Basler and G. Morata, 2002a Cells compete for decapentaplegic survival factor to prevent apoptosis in Drosophila wing development. Nature 416: 755-759.

Moreno, E., M. Yan and K. Basler, 2002b Evolution of TNF signaling mechanisms: JNKdependent apoptosis triggered by Eiger, the Drosophila homolog of the TNF superfamily. Curr Biol 12: 1263-1268.

Nagata, R., M. Nakamura, Y. Sanaki and T. Igaki, 2019 Cell Competition Is Driven by Autophagy. Dev Cell 51: 99-112 e114.

Nagata, S., 1997 Apoptosis by death factor. Cell 88: 355-365.

Narasimamurthy, R., P. Geuking, K. Ingold, L. Willen, P. Schneider et al., 2009 Structurefunction analysis of Eiger, the Drosophila TNF homolog. Cell Res 19: 392-394.

Ohsawa, S., K. Sugimura, K. Takino, T. Xu, A. Miyawaki et al., 2011 Elimination of oncogenic neighbors by JNK-mediated engulfment in Drosophila. Dev Cell 20: 315-328.

Paquette, N., M. Broemer, K. Aggarwal, L. Chen, M. Husson et al., 2010 Caspasemediated cleavage, IAP binding, and ubiquitination: linking three mechanisms crucial for Drosophila NF-kappaB signaling. Mol Cell 37: 172-182.

Park, J. M., H. Brady, M. G. Ruocco, H. Sun, D. Williams et al., 2004 Targeting of TAK1 by the NF-kappa B protein Relish regulates the JNK-mediated immune response in Drosophila. Genes Dev 18: 584-594.

Riesgo-Escovar, J. R., M. Jenni, A. Fritz and E. Hafen, 1996 The Drosophila Jun-Nterminal kinase is required for cell morphogenesis but not for DJun-dependent cell fate specification in the eye. Genes Dev 10: 2759-2768.

Ruan, W., N. Unsain, J. Desbarats, E. A. Fon and P. A. Barker, 2013 Wengen, the sole tumour necrosis factor receptor in Drosophila, collaborates with moesin to control photoreceptor axon targeting during development. PLoS One 8: e60091.

Sanchez, J. A., D. Mesquita, M. C. Ingaramo, F. Ariel, M. Milan et al., 2019 Eiger/TNFalpha-mediated Dilp8 and ROS production coordinate intra-organ growth in Drosophila. PLoS Genet 15: e1008133.

Shen, B., H. Liu, E. Y. Skolnik and J. L. Manley, 2001 Physical and functional interactions between Drosophila TRAF2 and Pelle kinase contribute to Dorsal activation. Proc Natl Acad Sci U S A 98: 8596-8601.

Silverman, N., R. Zhou, R. L. Erlich, M. Hunter, E. Bernstein et al., 2003 Immune activation of NF-kappaB and JNK requires Drosophila TAK1. J Biol Chem 278: 48928-48934.

Sluss, H. K., Z. Han, T. Barrett, D. C. Goberdhan, C. Wilson et al., 1996 A JNK signal transduction pathway that mediates morphogenesis and an immune response in Drosophila. Genes Dev 10: 2745-2758.

Stathopoulos, A., M. Van Drenth, A. Erives, M. Markstein and M. Levine, 2002 Wholegenome analysis of dorsal-ventral patterning in the Drosophila embryo. Cell 111: 687-701.

Stoven, S., I. Ando, L. Kadalayil, Y. Engstrom and D. Hultmark, 2000 Activation of the Drosophila NF-kappaB factor Relish by rapid endoproteolytic cleavage. EMBO Rep 1: 347-352.

Stoven, S., N. Silverman, A. Junell, M. Hedengren-Olcott, D. Erturk et al., 2003 Caspasemediated processing of the Drosophila NF-kappaB factor Relish. Proc Natl Acad Sci U S A 100: 5991-5996.

Stronach, B., A. L. Lennox and R. A. Garlena, 2014 Domain specificity of MAP3K family members, MLK and Tak1, for JNK signaling in Drosophila. Genetics 197: 497-513.

Tyler, D. M., W. Li, N. Zhuo, B. Pellock and N. E. Baker, 2007 Genes affecting cell competition in Drosophila. Genetics 175: 643-657. 
Vidal, S., R. S. Khush, F. Leulier, P. Tzou, M. Nakamura et al., 2001 Mutations in the Drosophila dTAK1 gene reveal a conserved function for MAPKKKs in the control of rel/NF-kappaB-dependent innate immune responses. Genes Dev 15: 19001912.

Wu, C., C. Chen, J. Dai, F. Zhang, Y. Chen et al., 2015 Toll pathway modulates TNFinduced JNK-dependent cell death in Drosophila. Open Biol 5: 140171.

Wu, D. C., and L. A. Johnston, 2010 Control of wing size and proportions by Drosophila myc. Genetics 184: 199-211.

Zhou, R., N. Silverman, M. Hong, D. S. Liao, Y. Chung et al., 2005 The role of ubiquitination in Drosophila innate immunity. J Biol Chem 280: 34048-34055.

\section{FIGURE LEGENDS}

Figure 1. eiger expression is induced in loser cells and is required for their competitive elimination.

A). Expression of egr mRNA and Traf4 mRNA are upregulated in WT loser cells, but is unchanged in Myc winner cells. Quantitative RT PCR on RNA from loser cells and Myc winner cells FAC-sorted from wing discs. Data are from one representative experiment and are normalized to actin5C.

B). Control, GFP-expressing clones induced in wing discs and allowed to grow for 24 hrs. B'). a-Cas-3 staining.

C). Clonal expression of egr for $24 \mathrm{hrs}$ leads to caspase activity (red) and rapid elimination of clones from wing discs; C'). a-Cas-3 staining.

D-E). Cell competition assay. D). Non-competitive control clones are marked with GFP and allowed to grow for defined periods of time (typically $48 \mathrm{hrs}$ ), after which measurement of clone size yields information on clonal growth. E). WT clones are generated in a background in which cells express an extra copy of Myc, yielding competitive interactions between the clones and surrounding Myc expressing cells. See Methods for details.

F). The egr ${ }^{3 A G}$ null allele prevents the elimination of loser cells. Clones grew for $48+/-4$ hrs.

G). Expression of egr-RNAi in loser cells prevents their competitive loss. Clones grew for 48 +/- 4 hrs. Error bars in F and G show mean and SD. Statistical significance was determined by unpaired t-tests using Welch's correction ${ }^{* * * *} p<0.0001$, ${ }^{* * *} p<0.001$, ns $=$ not significant. 
Figure 2. The TNFR Grnd contributes to the elimination of loser cells.

A). wgn mRNA is expressed primarily in the wing pouch (WP) region of the wing disc (96hr AEL).

B). Control clones grow to smaller sizes in $w g n^{22}$ compared to WT control clones; however, loser clone size in $w g n^{22}$ discs is similar to that its control clones, and also to WT loser clones. B'). The areas of the WP region in discs scored in B were not significantly different from each other. Clones grew for $48+/-4 \mathrm{hrs}$. Cell clones in wing discs from male larvae $\left(W T / Y\right.$ and $w g n^{22} / Y$ ) were measured. Error bars show mean and SD.

C). The Grnd/TNFR protein is uniformly expressed and apically localized in wing disc cells (disc is $120 \mathrm{hr}$ AEL; a maximum projection of a z-series is shown). C'). Crosssection showing apical Grnd localization in wing disc with GFP-marked WT loser clone (in tub>myc>Gal4 background).

D). Loss of $g r n d$ (using the grnd null mutants $\left(g r n d^{D f} / g r n d^{M i}\right)$ significantly reduces the elimination of loser cells under competitive conditions, but does not alter control clones in a non-competitive context. E). Clones grew for 48 +/- 3hrs. Error bars represent mean and SD. Statistical significance was determined by unpaired t-tests using Welch's correction. ${ }^{* * * *} \mathrm{p}<0.0001,{ }^{* *} \mathrm{p}<0.001,{ }^{*} \mathrm{p}<0.05$, ns $=$ not significant.

\section{Figure 3. Intracellular signaling by Grnd induces cell death, and Traf4 and Traf6 contribute to elimination of loser cells.}

A). Expression of $g r n d^{\text {extra }}$ in the wing pouch induces a small number of Cas3-positive cells (A').

B). Non-competitive control clones co-expressing GFP and grndextra.

C). Loser clones in the competitive context co-expressing GFP and grnd $^{\text {extra }}$.

D). Expression of grnd $^{\text {intra }}$ induces massive cell death and reduces overall wing disc size (D').

E, F). Expression of grnd $^{\text {intra }}$ in either control (E) and loser (F) clones results in their elimination from the disc within 48hr. Insets show the presence of the GFP-marked clones composed of 2-3 cells in each genotype at $24 \mathrm{hr} \mathrm{ACl}$.

G). Expression of $T r a f 4^{I R}$ specifically in loser cells prevents their elimination. Traf4 ${ }^{I R}$ expression in control clones does not alter their growth.

H). Elimination of loser cells is also suppressed in the Traf4 ${ }^{e x 1}$ null mutant background. 
I). Loss of Traf6 by expression of Traf6-RNAi in the loser cells prevents their elimination. All clones grew from 48-96hr +/- 4hr AEL. Error bars show mean and SD. Statistical significance was determined by unpaired t-tests using Welch's correction. ${ }^{* * *} p<0.0001$, ${ }^{\star * *} p<0.001,{ }^{* *} p<0.01$, ns $=$ not significant.

Figure 4. JNK signaling has a minor role in the elimination of WT loser cells. A). Loss of Tak1 does not prevent cell competition. Two different null alleles of Tak1 were tested. Clones were scored in wing discs from male larvae (Tak1/Y and WT/Y). B-C). Expression of UAS-egr induces massive cell autonomous and non-cell autonomous cell death. UAS-Egr was co-expressed with UAS-GFP using DppGal4 in WT (B) or hep ${ }^{r 75}$ wing discs (C). Cas-3 (red) staining highlights dying cells. C). The hep ${ }^{r 75}$ mutation blocks the majority of apoptosis induced by UAS-egr. Wing discs are from $W T / Y$ males or hep ${ }^{r 75} / Y$ hemizygous males.

D). WT loser clones marked with GFP die at high frequency $24 \mathrm{hr}$ after clone induction (ACI). (D'). Cas-3 staining.

E). $24 \mathrm{hr} \mathrm{ACl}$, compared to $W T / Y$ loser clones, cell death in loser clones is reduced in the hep ${ }^{r 75} / Y$ background. (E'). Cas-3 staining.

F). Violin plots with quantification of Cas-3 (+) cells/clone at $24 \mathrm{hr} \mathrm{ACl}$. The percent clones with Cas-3 $(+)$ cells is shown at the top, and $n=$ number of clones scored per genotype (WT/Y and hep $\left.{ }^{r 75} / Y\right)$.

G). By $50 \mathrm{hr} \mathrm{ACl,} \mathrm{loser} \mathrm{clones} \mathrm{in} \mathrm{a} \mathrm{WT} \mathrm{background} \mathrm{are} \mathrm{significantly} \mathrm{smaller} \mathrm{than}$ controls, and $h e p^{r 75}$ does not prevent their competitive elimination. Clones were scored in wing discs from male larvae (WT/Y and hep $\left.{ }^{r 75} / Y\right)$.

H). Loser clones expressing UAS-Bsk ${ }^{D N}$ are larger than WT loser clones, but are still significantly smaller than the controls, suggesting that competition is only partially blocked.

Error bars in $(\mathbf{A}),(\mathbf{G})$ and $(\mathbf{H})$ show mean and standard deviation. Statistical significance was determined by unpaired t-tests for each condition compared to controls; Welch's correction was used for data in F-G. ${ }^{* * * *} p<0.0001,{ }^{* * *} p<0.001,{ }^{* *} p<0.01,{ }^{*} p<0.05$ relative to $y w$ controls.

Figure 5. Epistatic evidence for divergence of Grnd/TNFR signaling from JNK to the CCSM in the elimination of loser cells. 
A). Sibling $\operatorname{Tak}^{2}$ mutant male (left) and female (right) larvae clonally expressing cell UAS-grnd ${ }^{\text {intra }}$ under tubGal4 control. The complete loss of Tak1 in hemizygous males abolishes the massive death induction due to expression of Grndintra, while female Tak $1^{2} /+$ larvae remain susceptible to Grnd ${ }^{\text {intra }}$-mediated cell killing and the consequent overall size reduction.

B-D). Tak $1^{2}$ mutant prevents apoptosis induced by grnd ${ }^{\text {intra }}$ in non-competitive control clones, but not cell competition-induced apoptosis in loser clones. B). Control and loser clones from $W T / Y$ males. C). Clones expressing grnd ${ }^{\text {intra }}$ do not grow due to extensive cell death (see Fig. 3E-F) and are represented here as "no clones". D). Under noncompetitive conditions, $\operatorname{Tak}^{2} / Y$ prevents grnd ${ }^{\text {intra }}$ expression from killing cells in control clones (compare to (B, WT/Y Ctls), indicating that these cells die due to JNK-mediated signaling. $\operatorname{Tak}^{2} / Y$ does not prevent the elimination of loser clones in cell competition (compare to $\mathbf{B}, W T / Y$ losers), suggesting that JNK signaling is not required for their death. Clones grew for $48+/-3 h r s$. Error bars show mean and SD. Statistical significance was determined by t-tests using Welch's correction. ${ }^{* * *} p<0.0001,{ }^{* *} p<0.01$. E-H). Cell death induced by Dredd expression is epistatic to grnd. E). Clones of WT loser cells (Losers) clones are smaller that WT control clones (Ctls) due to their competitive elimination. F). Expression of Dredd in control clones induces cell death (MEYER et al.), reducing clone size (F Ctls vs E Ctls). In the competitive context, Dredd expression in loser cells enhances their elimination. G). Knock-down of grnd with UASgrnd-RNAi $\left(g_{r n d^{\prime R}}\right)$ expression specifically in loser clones efficiently suppresses their elimination (Losers), but does not alter the size of control clones (Ctls). H). Coexpression of Dredd and grnd $^{\prime R}$ does not suppress cell death induced by Dredd expression in non-competitive controls, nor does it suppress the elimination of loser cells. However, the enhancement of loser elimination by Dredd expression is reduced by grnd knockdown ( $\mathrm{p}=0.0049,+$ Dredd losers in $(\mathbf{G})$ vs grnd $^{I R}+$ Dredd losers in $(\mathbf{H})$; $\mathrm{p}=0.0715$, WT losers in $(\mathbf{E})$ vs grnd ${ }^{\prime R}+$ Dredd losers in $\mathbf{( H )}$. Clones were allowed to grow for $48+/$ - 3hrs. Error bars show mean and standard deviation. Statistical significance was determined by unpaired t-tests using Welch's correction. ${ }^{* * * *} p<0.0001,{ }^{* * *} p<0.001$, ${ }^{* *} p<0.01,{ }^{*} p<0.05$, ns $=$ not significant. 
Figure 1. A
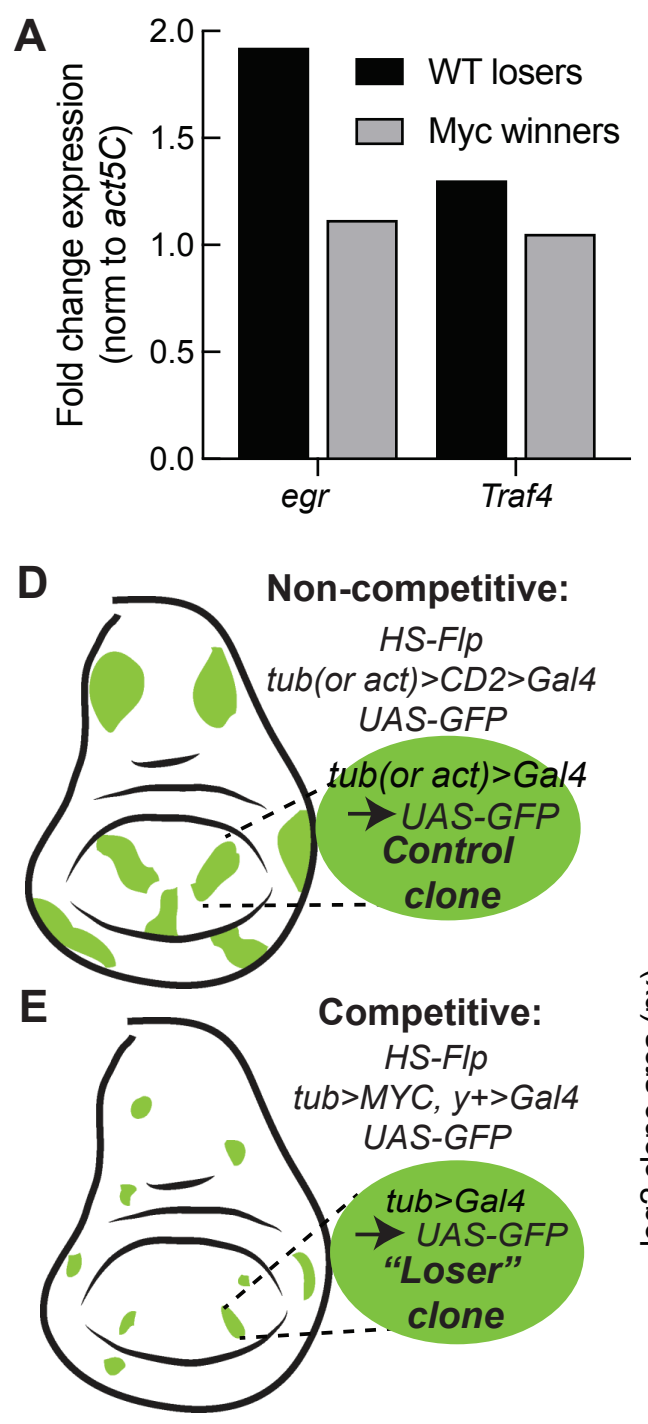

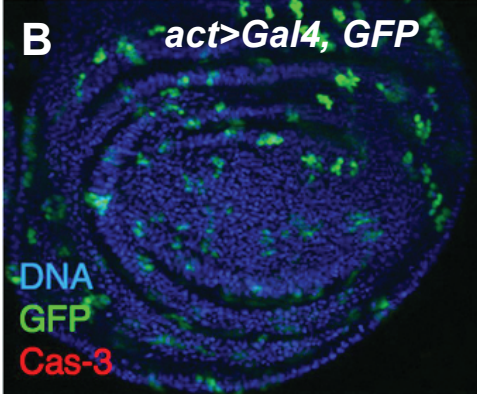

C, act>Gal4, egr; GFP

, ,., e t:
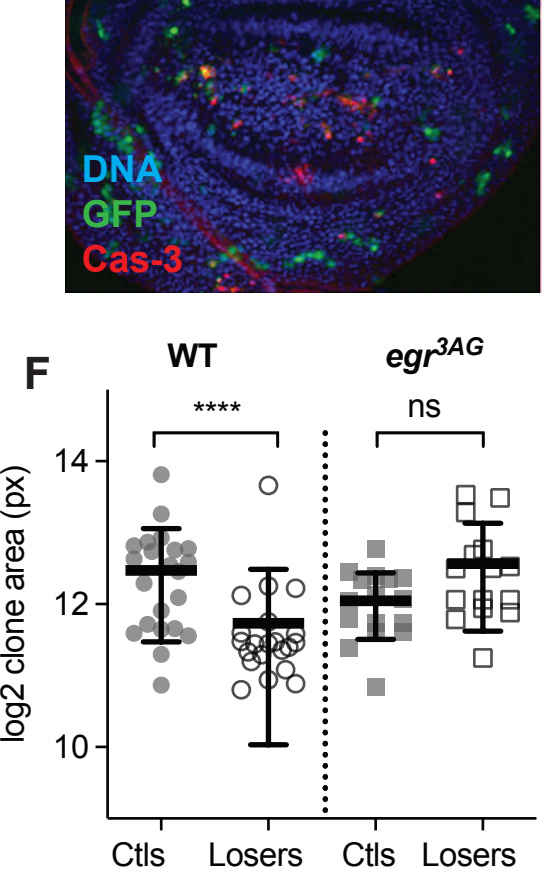
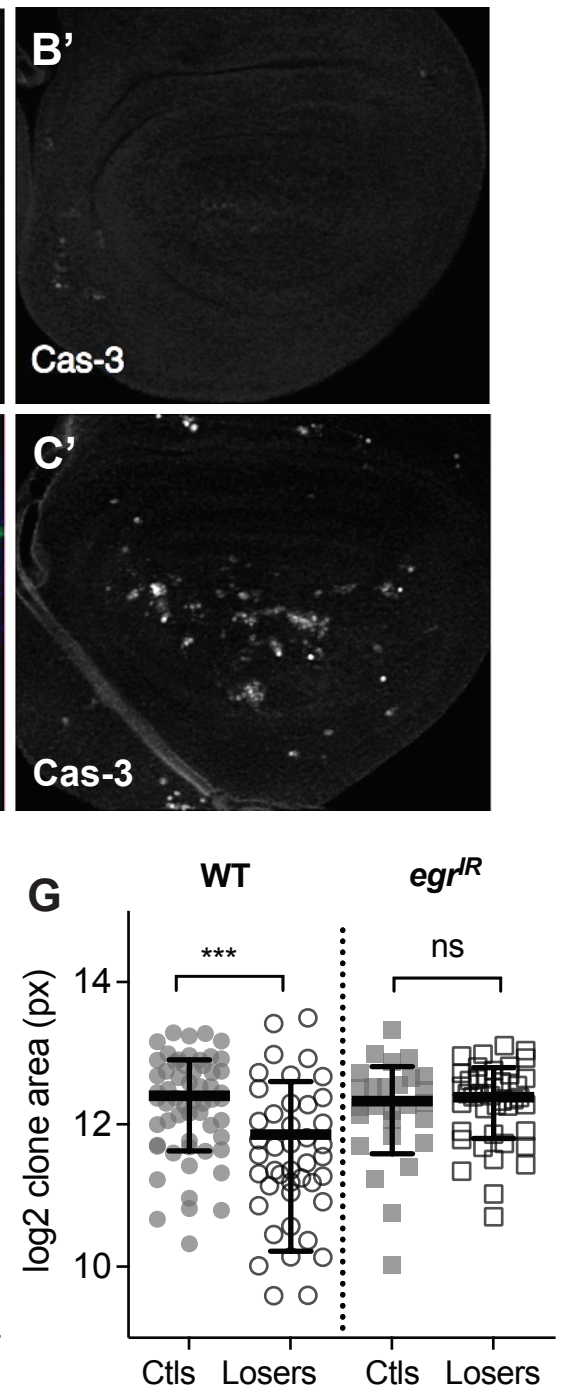
Figure 2.
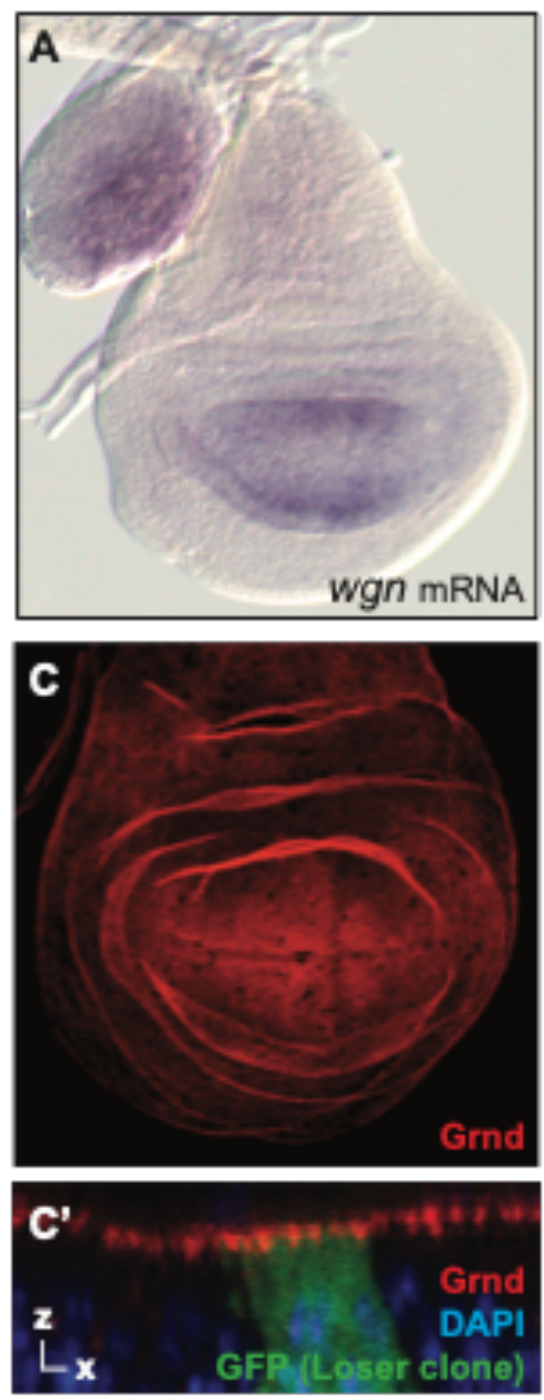

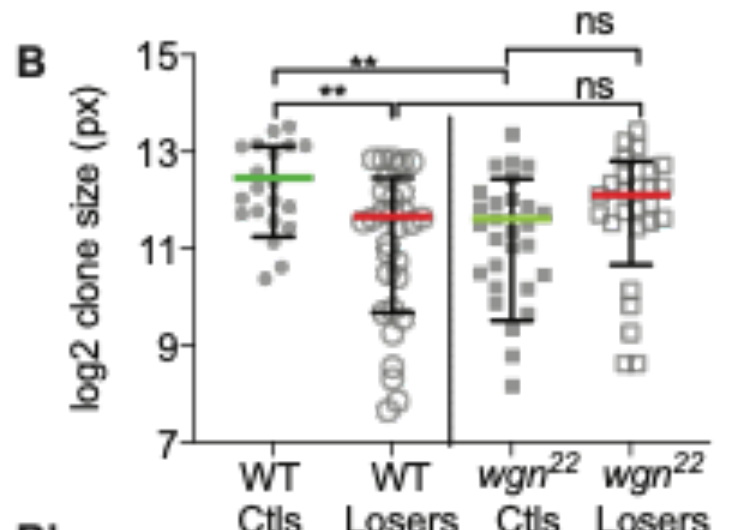

B'
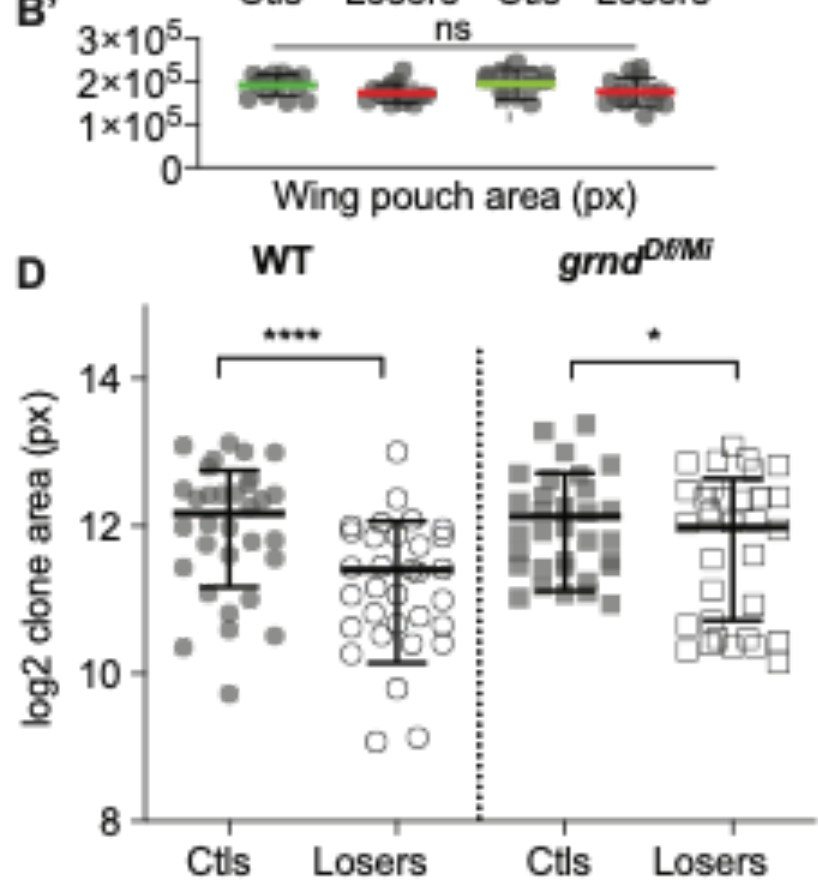
Figure 3.
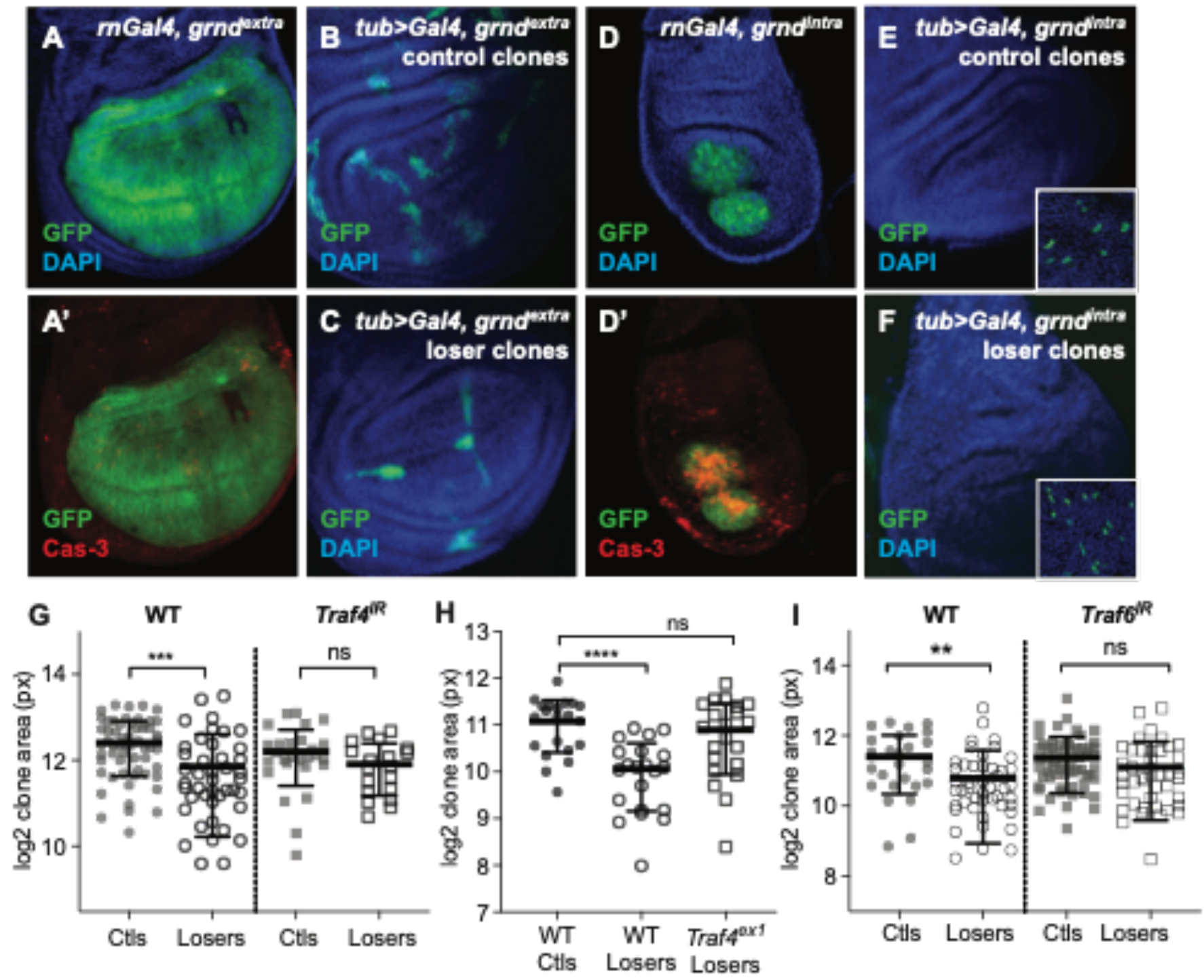
Figure 4.
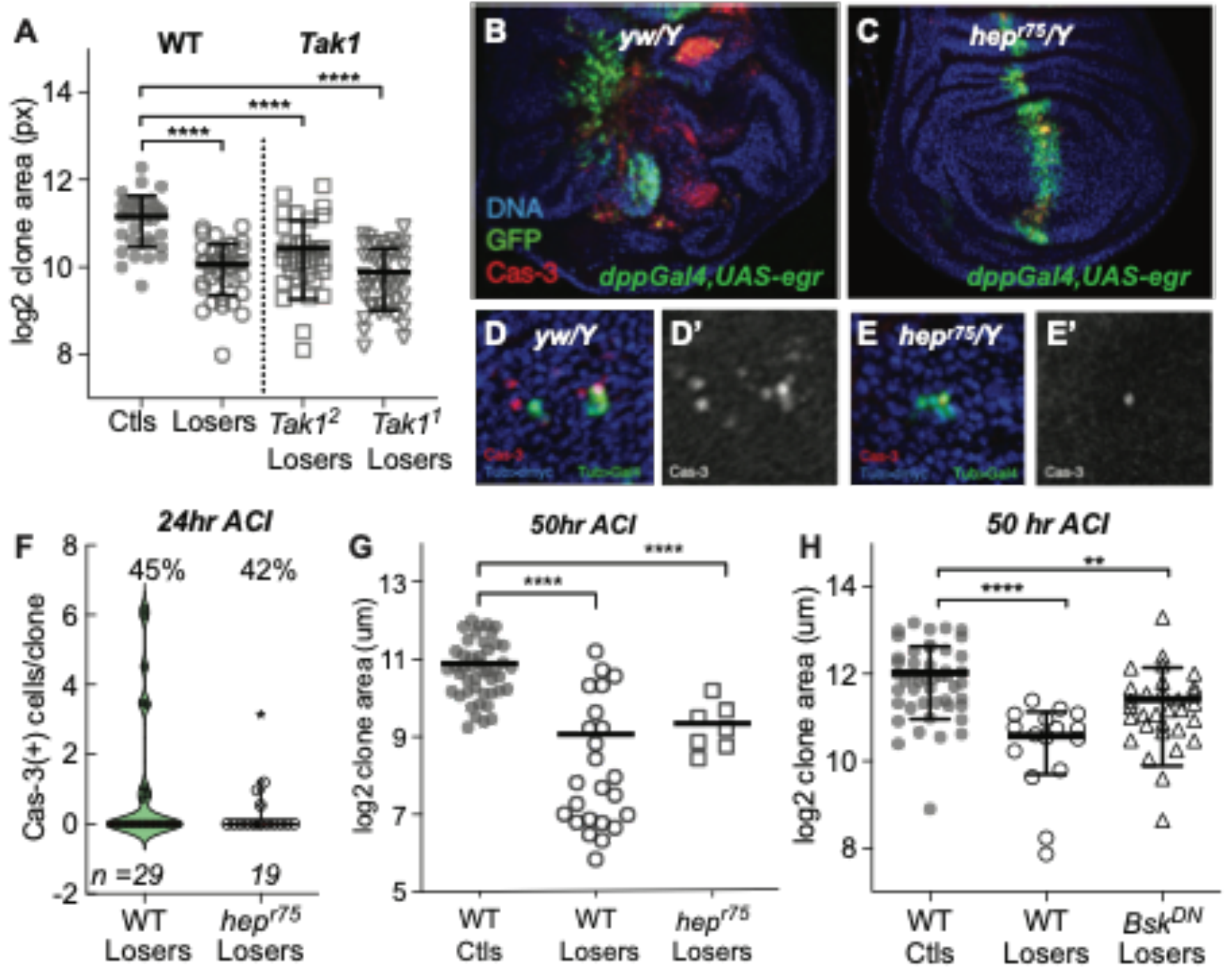
Figure 5.
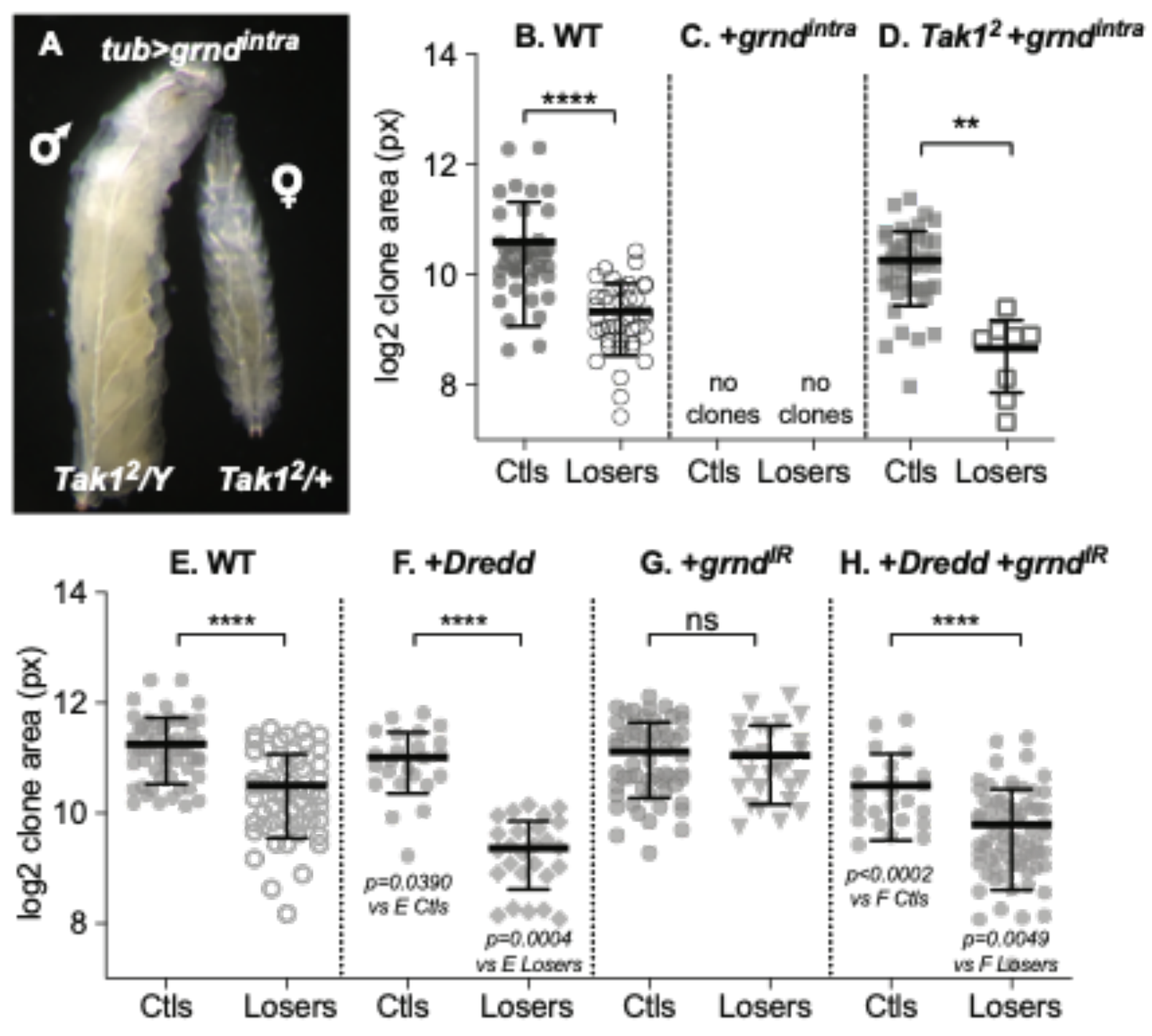